Document downloaded from:

http://hdl.handle.net/10251/28655

This paper must be cited as:

Lloret Soler, JA.; Ramos Pascual, F.; Sancho Durá, J.; Gasulla Mestre, I.; Sales Maicas, S.; Capmany Francoy, J.; Xue, W.... (2011). The Influence of Optical Filtering on the Noise Performance of Microwave Photonic Phase Shifters Based on SOAs. JOURNAL OF LIGHTWAVE TECHNOLOGY. 29(12):1746-1752. doi:10.1109/JLT.2011.2135839.

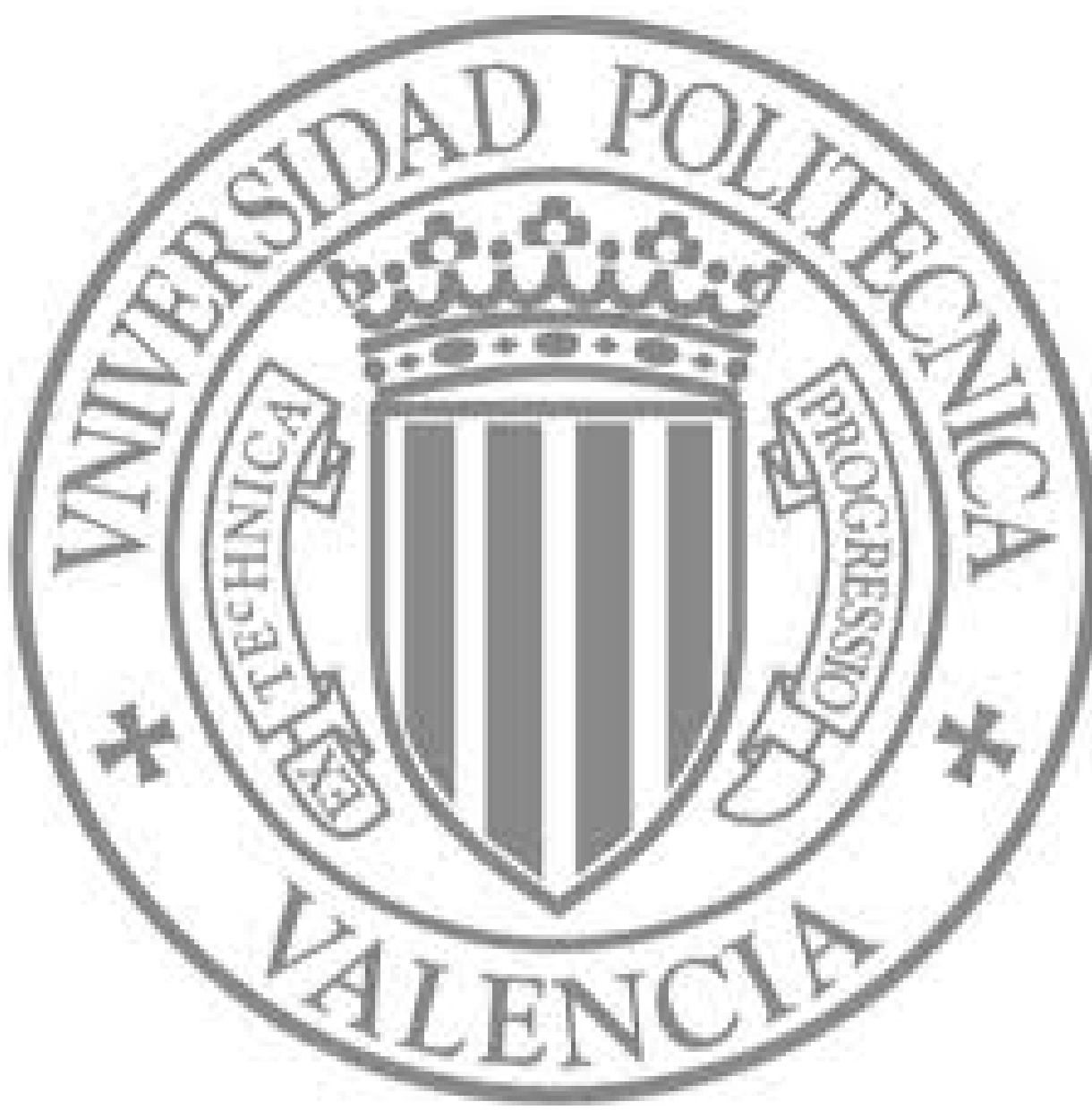

The final publication is available at

http://dx.doi.org/10.1109/JLT.2011.2135839

Copyright Institute of Electrical and Electronics Engineers (IEEE) 


\title{
The Influence of Optical Filtering on the Noise Performance of Microwave Photonic Phase Shifters based on SOAs
}

\author{
Juan Lloret, Francisco Ramos, Member, IEEE, Weiqi Xue, Juan Sancho, Ivana Gasulla, Salvador \\ Sales, Senior Member, IEEE, Jesper Mørk and José Capmany, Fellow, IEEE
}

\begin{abstract}
Different optical filtering scenarios involving microwave photonic phase shifters based on semiconductor optical amplifiers are investigated numerically as well as experimentally with respect to noise performance. Investigations on the role of the modulation depth and number of elements in cascaded shifting stages are also carried out. Suppression of the noise level by more than $5 \mathrm{~dB}$ has been achieved in schemes based on band-pass optical filtering when three phase shifting stages are cascaded.
\end{abstract}

Index Terms-Slow and Fast Light (SFL), Phase Shifter, Semiconductor Optical Amplifier (SOA), Noise Figure.

\section{INTRODUCTION}

$\mathrm{T}^{\mathrm{n}}$ HE capability of controlling the group velocity of light by exploiting novel techniques and materials has attracted increasing interest in recent years [1]-[2]. This discipline, which is referred to as slow and fast light (SFL), provides new opportunities for implementing a wide variety of signal processing functionalities.

A field of activity in which SFL becomes potentially interesting is microwave photonics (MWP) [3]. Among all the applications to be implemented, two are of particular importance: tunable filters [4] and reconfigurable phased array antennas [5]. To this end, broadband tunable microwave phase shifters and tunable true time delay lines represent key subsystems to be developed. Electronic devices cannot offer compact solutions for broadband frequency ranges [6]. Hybrid technologies based on ferroelectric and liquid crystal have the ability to realize large phase or time shifts [6]. However, the limited frequency range and the high power consumption, $\sim 100 \mathrm{~W}[6]$, have led to the consideration of alternative optical methods [7]. To date, several optical technologies have been reported in the literature, including those based on optical fibers [8], photonic crystals [9] and semiconductors [10].

So far, semiconductor based schemes appear as the most

Manuscript received November 3, 2010. This work was supported in part by the EU FP7 within project GOSPEL, the Excellency Award Programme GVA PROMETEO 2008/092 and the Plan Nacional I+D TEC 2007-68065C03-01.

J. Lloret, F. Ramos, J. Sancho, I. Gasulla, S. Sales and J. Capmany are with the iTEAM Research Institute, Universidad Politécnica de Valencia, 46022 Valencia, Spain (e-mail: jualloso@iteam.upv.es).

W. Xue and J. Mørk are with the DTU Fotonik, Department of Photonics Engineering, Technical University of Denmark, 2800 Kgs. Lyngby, Denmark (e-mail: wexu@fotonik.dtu.dk). promising technology since phase shifts up to $2 \pi$ radians have recently been achieved when cascading several photonic shifting stages (PSS) [11]. Moreover, the semiconductor technology is very mature, offers the potential of velocity control by means of both electrical and optical external signals and can be easily integrated.

The physical process governing the SFL operating regime in SOAs is coherent population oscillations (CPO), which leads to four-wave mixing (FWM) and complex cross-gain modulation (XGM) [12]. The CPO effect relies on temporal gain and refractive index gratings, which are responsible for the phase shift of the modulating microwave signal [12]. Nevertheless, if both sidebands are detected after the SOA, the refractive index dynamics is cancelled out, limiting the total phase shift to a few tens of degrees over a narrow bandwidth [13]. To overcome the above-mentioned limitation, the phase shift can be enhanced by filtering out the long wavelength sideband prior to photodetection [14]. The main drawback, however, is that the radiofrequency (RF) subcarrier is attenuated to a larger extent than the noise, and the final signal-to-noise ratio (SNR) is deteriorated; even considering that the amplified spontaneous emission (ASE) noise is also filtered out [15].

From the microwave link point of view, several figures of merit must be evaluated to give objective data regarding its performance [16]. In particular, the performance should be studied, at least, in terms of RF net gain, noise figure [15], [17]-[18] and nonlinear distortion leading to generation of harmonic and intermodulation components [19]-[20].

To date, several investigations have already identified the intensity noise as the dominant noise contribution at the output of SOA-based MWP phase shifters [15], [17]-[18]. The purpose of this paper is to evaluate the output noise level, as well as the output SNR of MWP phase shifters based on SOAs. The paper is organized as follows. In Section II, the model for calculating the relative intensity noise (RIN) at the output of the structure is presented. In Section III, the experimental setups involving three different optical filtering scenarios are detailed. Both the numerical and the experimental results of the RIN as a function of operation parameters can be found in Section IV. The most important discussions related to the results, as well as some considerations regarding the impact of cascading several PSSs are reported in Section V. Finally, Section VI summarizes the main conclusions. 


\section{THEORY}

A numerical model for the purpose of calculating the RIN spectral density of SOA-based MWP phase shifters is presented. In absence of modulation, a semi-classical description already published in the literature can be used to evaluate the noise properties at the SOA output [21]. The model considers that the noise effect at the output of the semiconductor waveguide can be treated as a small perturbation, which is superimposed on the output mean optical power. Therefore, the optical power at the SOA output can be expressed as

$$
P(z=L, t)=\bar{P}(z=L)+\partial P(z=L, t),
$$

where $\bar{P}$ and $\partial P$ denote the mean output optical power and the fluctuating power due to noise, respectively. $z$ and $t$ are the longitudinal and temporal coordinates respectively, and $L$ is the active cavity length.

Building on this, the RIN can be defined as:

$$
R I N(\omega)=\frac{P S D\{\partial P\}}{\bar{P}^{2}}=\frac{\mathcal{F}\left\{R_{\partial P, \partial P}\right\}}{\bar{P}^{2}},
$$

with $\omega, P S D, \mathcal{F}$ and $R$ being the optical frequency, the power spectral density, the Fourier transform and the correlation function, respectively.

On the other hand, the total RIN at the SOA output can be written as the sum of four contributions by developing (2) in terms of electrical field [21]:

$$
R I N(\omega)=R_{0}(\omega)+R_{s p}(\omega)+R_{g}(\omega)+R_{g, s p}(\omega) .
$$

The four terms appearing on the right-hand side of (3) describe the noise contributions of the injected signal, the spontaneous emission, the carrier noise and the carrierspontaneous beat-note, respectively.

As already mentioned, the total microwave phase shift can be enhanced by filtering out the lower frequency modulation sideband prior to photodetection but this filtering process must be carefully taken into account when calculating the output noise spectrum.

Hence, at the output of the optical filter, the total RIN density becomes

$$
\operatorname{RIN}^{f}(\omega)=\operatorname{RIN}(\omega)|H(\omega)|^{2} .
$$

In (4), the index $f$ indicates "filtered" and $H(\omega)$ corresponds to the frequency transfer function of the optical filter. It is important to remark that (4) can support all kinds of linear filtering schemes by properly choosing the corresponding transfer function. In particular, $|H(\omega)|^{2}=1$ when no filtering is performed.

The photodetected RIN power is defined as

$$
\sigma_{R I N}^{2}(\omega)=\left(\Re P_{p h}\right)^{2} r_{I}^{2}(\omega)
$$

with $\mathfrak{R}$ and $P_{p h}$ being the responsivity and the input optical power to the photodetector, respectively. Because of previous filtering, $r_{I}^{2}$ is characterized by an asymmetric spectrum which is defined as [17]:

$$
\begin{aligned}
r_{I}^{2}(\omega)=\int_{-\omega-\frac{\Delta \omega}{2}}^{-\omega+\frac{\Delta \omega}{2}} & R I N^{f}\left(\omega^{\prime}\right) d \omega^{\prime} \\
& +\int_{\omega-\frac{\Delta \omega}{2}}^{\omega+\frac{\Delta \omega}{2}} \operatorname{RIN}^{f}\left(\omega^{\prime}\right) d \omega^{\prime} .
\end{aligned}
$$

Where $\Delta \omega \rightarrow 0$ accounts for the resolution bandwidth.

Finally, the output SNR of the phase shifting element can be expressed as

$$
S N R_{\text {out }}=\frac{C_{\text {out }}}{\int_{\omega_{0}-\frac{B W}{2}}^{\omega_{0}+\frac{B W}{2}} \sigma_{R I N}^{2}(\omega) d \omega}
$$

where $C_{\text {out }}, \sigma_{R I N}^{2}, B W$ and $\omega_{0}$ are the electrical output carrier peak power, the power density due to RIN expressed as a function of $1 / \mathrm{Hz}$, the system bandwidth and the RF tone frequency, respectively. Therefore, it must be noticed that the total $S N R_{\text {out }}$ is application-dependent.

\section{EXPERIMENTAL SETUP}

In Fig. 1(a), the basic set-up showing the configurations which have been investigated is sketched. The goal of the study is to discern the filtering scenario which leads to optimum noise performance of the phase shifting microwave link.

The set-up basically consists of a distributed feedback (DFB) laser, which can (D) or cannot (S) be modulated by means of a Mach-Zehnder modulator (MZM). A variable attenuator at the erbium doped fiber amplifier (EDFA) input is used to adjust the proper optical power at the SOA input. Both the input and the output of the EDFA and the SOA are appropriately isolated. At the SOA output, three kinds of optical filtering schemes can be employed: (a) no filtering, (b) notch filtering, and (c), band-pass filtering. In order to avoid any effect of the photodetector (PD) on the noise measurements, a variable attenuator is inserted to maintain a fixed power level at the PD input. Finally, the electrical spectrum is measured using an electrical spectrum analyzer (ESA).

The optical notch filter (ONF) is implemented by means of a fiber Bragg grating (FBG) operating in transmission, whose normalized amplitude and phase transfer functions are shown in Fig. 1(b). The grating provides attenuation greater than 32 $\mathrm{dB}$ at the frequency where the notch is centered and has a -3 $\mathrm{dB}$ bandwidth of approximately $9 \mathrm{GHz}$. As seen, the FBG displays dispersive behavior at frequencies belonging to both slopes of the amplitude transfer function. 


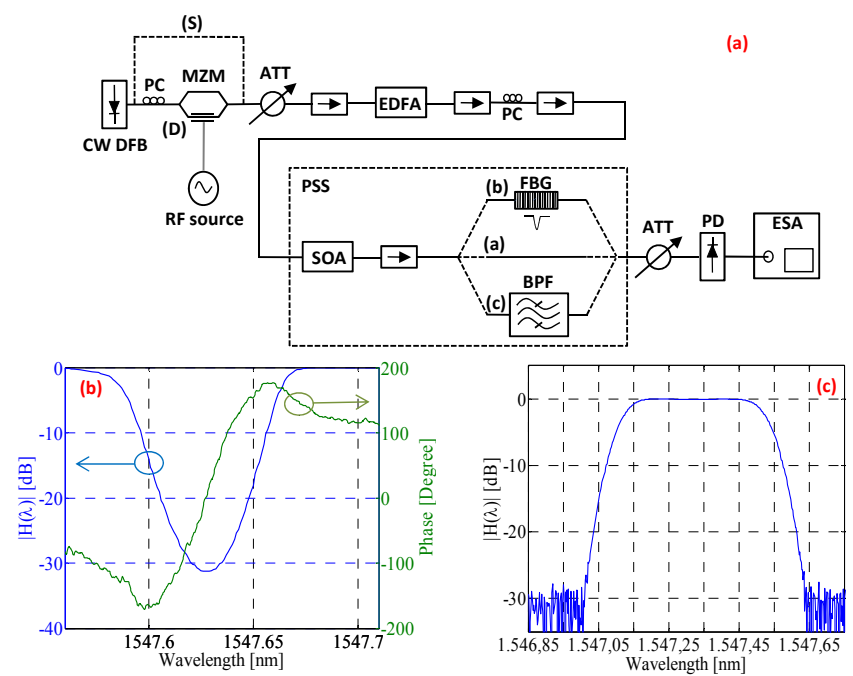

Fig. 1. (a) Experimental set-up. (b) Measured normalized amplitude and phase transfer function of the notch filter (FBG). (c) Measured normalized amplitude transfer function of the band-pass filter (BPF). The emission wavelength of the CW DFB laser is $1547.51 \mathrm{~nm}$.

Fig. 1(c) shows the amplitude transfer function of the bandpass filter (BPF). The filter is characterized by zero dispersion, maximum attenuation up to $30 \mathrm{~dB}$, and a $-3 \mathrm{~dB}$ bandwidth around $53 \mathrm{GHz}$.

\section{RESULTS}

\section{A. Static behavior}

In this section, numerical and experimental results for the static characteristics of the structure are presented; i.e. for the case without an applied microwave signal (see Fig. 1(a), configuration marked as (S)). The system parameters have been set in order to induce a phase shift of approximately $150^{\circ}$ on a RF tone placed at $15 \mathrm{GHz}$. The parameters have been chosen in accordance with previous experiments [14]. The worst scenario in terms of noise at the output of the phase shifting element occurs when the SOA displays the maximum gain, caused by the maximum injection current. For all the measurements and calculations, the current of $350 \mathrm{~mA}$ has been chosen. Other relevant system parameters used in the numerical calculations can be found in Table I.

Fig. 2 shows the RIN at the output of the phase shifting element when considering the three different filtering scenarios. Experimental results are compared with calculations obtained using the numerical model described in Section II.

Considering the case where no filtering is performed, one observes that at high frequencies the noise level is nearly flat, effect which is in good agreement with the wideband nature of the spontaneous emission process. However, at low frequencies, a dip in power appears and the spectrum displays a spectral hole, showing the typical high-pass noise filtering behavior of the SOA [21]. The numerical and experimental results are seen to agree very well.

Next, we consider the influence of an optical notch filter. The spectral detuning between the optical carrier and the notch position of the FBG is adjusted to $15 \mathrm{GHz}$, as clearly seen in
TABLE I

MAIN SYSTEM PARAMETERS

\begin{tabular}{ccc}
\hline \hline Symbol & Definition & Value \\
\hline$L$ & active region length & $1 \mathrm{~mm}$ \\
$t$ & active region thickness & $100 \mathrm{~nm}$ \\
$\omega$ & active region width & $2.5 \mu \mathrm{m}$ \\
$\alpha$ & linewidth enhancement factor & 6 \\
$\Gamma$ & confinement factor & 0.23 \\
$\gamma$ & unsaturable loss & $4000 \mathrm{~m}^{-1}$ \\
$\tau$ & carrier lifetime & $210 \mathrm{ps}$ \\
$a$ & differential gain & $1.1 \cdot 10^{-20} \mathrm{~m}^{2}$ \\
$N_{t}$ & carrier density at transparency & $1 \cdot 10^{24} \mathrm{~m}^{-3}$ \\
$P_{\text {out,sat }}$ & saturation output power & $5 \mathrm{dBm}$ \\
$I$ & injection current & $350 \mathrm{~mA}$ \\
$P_{\text {in }}$ & input optical power to the SOA & $-1 \mathrm{dBm}$ \\
\hline \hline
\end{tabular}

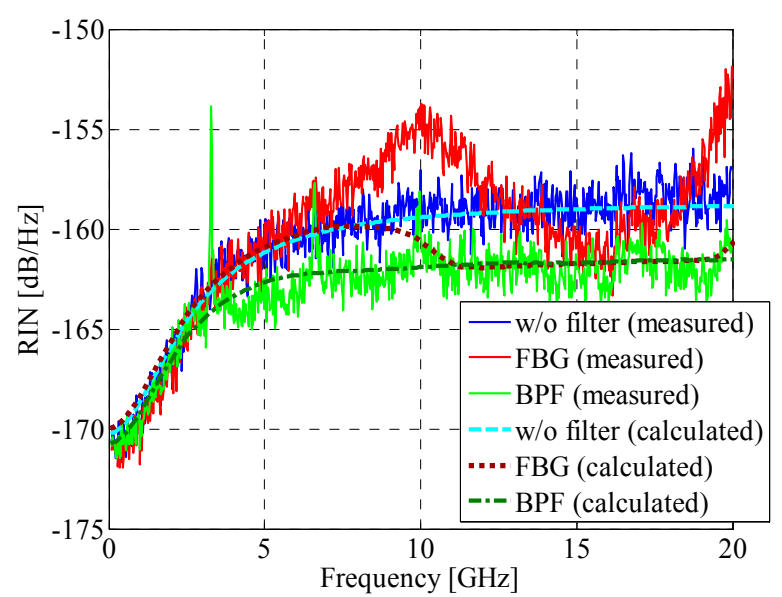

Fig. 2. Measured and calculated RIN spectral density for the three different filtering scenarios.

the spectrum. A RIN enhancement is observed around 10 and $20 \mathrm{GHz}$. This effect originates from FM-AM-noise conversion mediated by the dispersive characteristic of the FBG and will be discussed later [22]-[23]. As it has been already demonstrated, the dispersive nature of the output filter plays a crucial role to accurately model the phase feature of SOAbased MWP phase shifters [24]. Different noise contributions dominate in different frequency ranges. In particular, for frequencies lower than $\sim 5 \mathrm{GHz}$ the ASE noise dominates, whereas at larger frequencies, the noise contribution originating from the FM-AM-noise conversion becomes the most significant. These frequency ranges obviously depends on the spectral detuning between the optical carrier and the FBG notch. There is a disagreement between calculated and experimental data, because the FM-AM-noise conversion phenomenon caused by the FBG is not included in the numerical model. The dispersion due to the FBG is zero at the notch central frequency, so the numerical model yields a reliable result at $15 \mathrm{GHz}$ in this case.

Regarding the case in which the BPF is used, the RIN level is lowered because of noise filtering. Before, when no filter or the FBG were considered, the noise was integrated over the bandwidth of the PD (around $75 \mathrm{GHz}$ ). However, if the BPF is inserted prior to photodetection, the integration bandwidth is limited to that imposed by the filter, which is approximately 


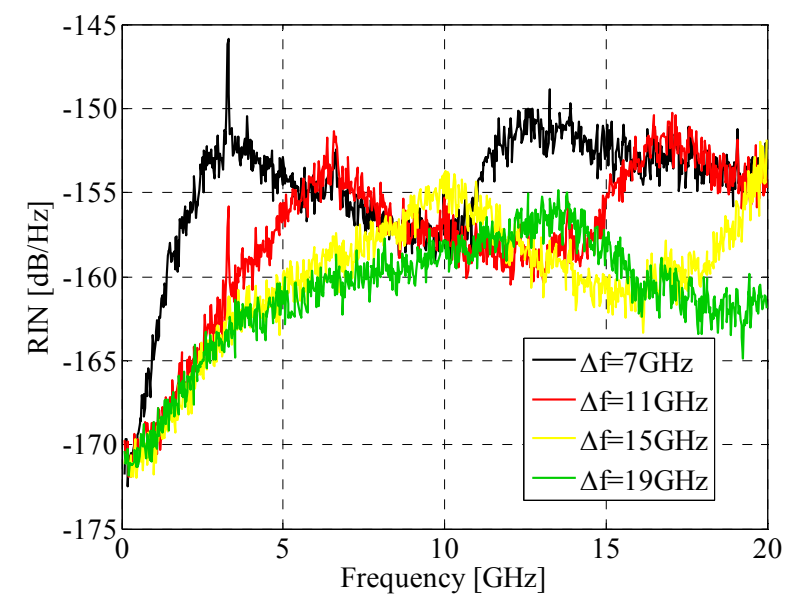

Fig. 3. Measured RIN spectrum with different degrees of FM-AM-noise conversion according to the spectral spacing $(\Delta \mathrm{f})$ between the optical carrier and the notch placement.

$53 \mathrm{GHz}$. That is the reason why, in this case, the RIN level decreases close to $3 \mathrm{~dB}$ compared to the no filter case. The RIN level is at last imposed by the frequency of the RF tone, since a filter as narrow as that frequency could be used. Numerical calculations are in perfect agreement with the experimental results.

In order to get a deeper insight into the implications of the FBG dispersion on the RIN performance, studies have been carried out by varying the relative position between the optical carrier and the notch spectral placement. The results are given in Fig. 3. They show that the efficiency of the FM-AM-noise conversion is frequency-dependent according to the phase transfer feature of the dispersive element. Specifically, the maximum conversion efficiency, and thereby the largest RIN enhancement, occurs when the most dispersive spectral region of the FBG characteristic coincides with the carrier frequency. A peak can also be observed at the frequency of $3.5 \mathrm{GHz}$ approximately, which corresponds to the relaxation oscillation frequency of the DFB laser source used to pump the EDFA. It also must be noticed that the larger the frequency detuning $\Delta \mathrm{f}$, the lower the RIN level at the frequency where the notch is centered, because the conversion efficiency becomes lower.

\section{B. Dynamic behavior}

This section considers the dynamic operating regime of the phase shifting element, i.e. the microwave signal is taken into account (see Fig. 1(a), configuration marked as (D)).

There is a trade-off between SNR and distortion when choosing the optical modulation depth, $q$, defined in [25], in SOA-based MWP phase shifters. Small-signal modulation, with $q$ usually below $0.5 \%$, is employed to alleviate the distortion and to yield good enough output SNR.

Fig. 4 shows the numerical and measured RIN spectral density as a function of the filtering scheme and $q$. It must be pointed out that whatever the $q$ the noise level around $15 \mathrm{GHz}$ keeps practically constant. This observation justifies the use of a static numerical model to predict the RIN when considering the microwave tone. Perfect agreement between experimental
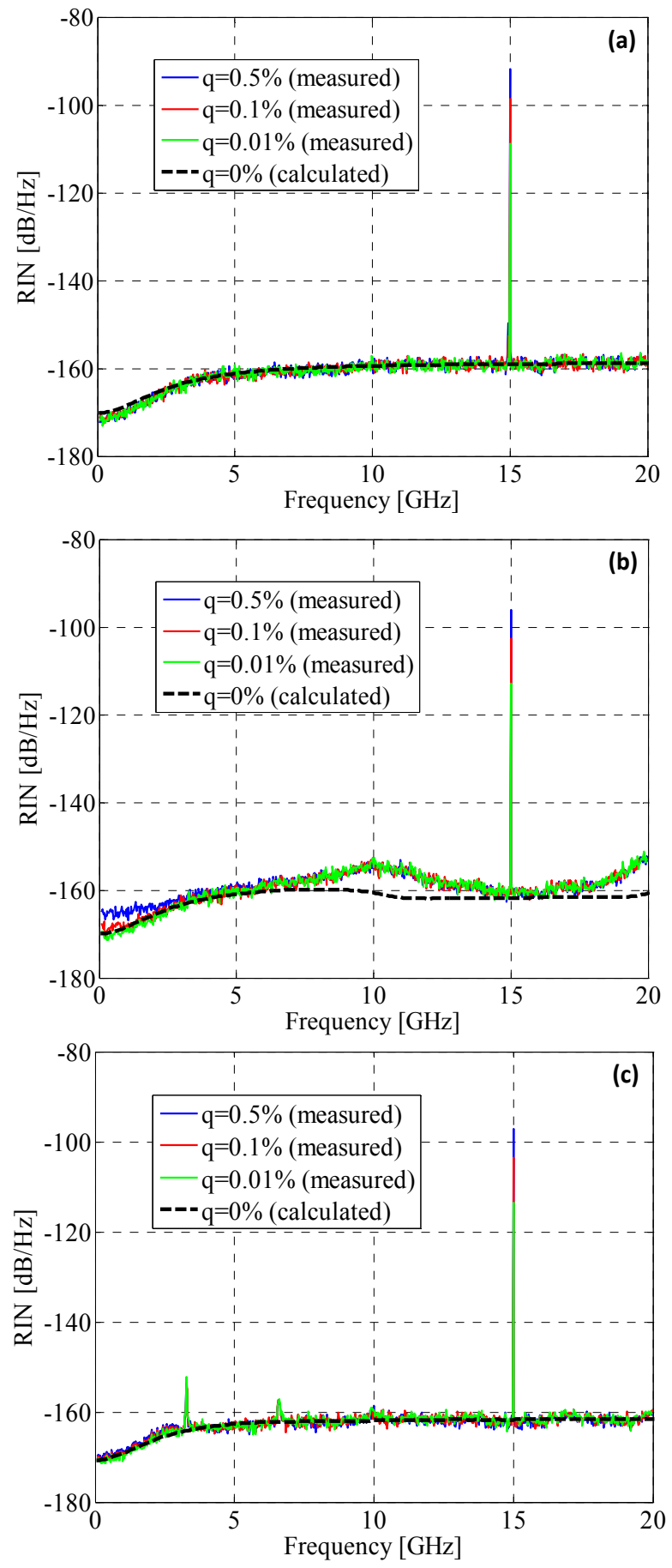

Fig. 4. Calculated RIN spectral density as a function of the $q$ when: (a) no optical filtering is considered, (b) when using FBG and (c) when using BPF.

and calculated results is obtained in the scenario without optical filter, as shown in Fig. 4(a). On the other hand, when the FBG is used for filtering, numerical and measured results do not agree due to the FM-AM-noise conversion. However, numerical data is roughly consistent with experimental results at $15 \mathrm{GHz}$ because zero dispersion is induced at the notch frequency (see Fig. 4(b)). The degree of saturation of the SOA has a major impact on the RIN spectrum, in particular at low frequencies, where a RIN enhancement can be seen as a 


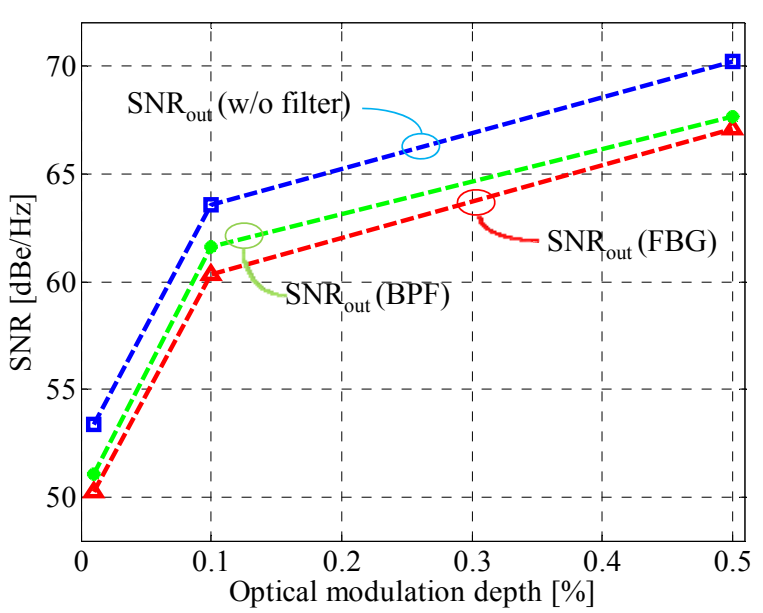

Fig. 5. SNR of the microwave link element as a function of $q$ and the optical filtering scenario.

function of $q$. The dip in power depends on the optical power and the $q$, because it results from interactions of both the optical carrier and the modulation sidebands with the noise. Consequently, the larger the $q$, the larger the RIN enhancement. This fact justifies the discrepancies at low frequencies between experimental and numerical results, due to the static nature of the model. Finally, when considering the BPF-based scheme, Fig. 4(c), the fitting between calculated and measured data is perfect as a function of the frequency.

\section{DISCUSSION}

\section{A. SNR evaluation}

The SNR is obtained as a function of $q$ to evaluate the noise properties of the microwave link. The SNR is evaluated for the three different filtering schemes to discern which yields the better noise performance. To grant the results independence to the system bandwidth, the SNR has been obtained in terms of $\mathrm{dB} / \mathrm{Hz}$.

Fig. 5 shows the output SNR of the phase shifting element. It must be pointed out that the larger the $q$, the higher the $\mathrm{SNR}$. The largest $\mathrm{SNR}_{\text {out }}$ is obtained when no filter is inserted prior to photodetection. In this case, both modulation sidebands are photodetected, giving as a result the maximum RF power. However, the induced phase shift on the microwave signal is lower by an order of magnitude when optical filtering is not applied [14]. When using optical filtering before the photodetection, the left frequency modulation sideband is suppressed. Although the intensity noise is also lowered, the left frequency sideband is attenuated to a larger extent and the final SNR is diminished. For filtering by a BPF, a larger amount of noise is rejected than the case of FBG filtering and the SNR performance is improved.

\section{B. Multi-PSS-based MWP phase shifter}

The cascade of PSSs is a solution suitable for improving the total phase shift of the microwave link. By using a proper configuration based on three PSSs, continuous tunable phase shift of $360^{\circ}$ can be achieved [11]. The use of regeneration

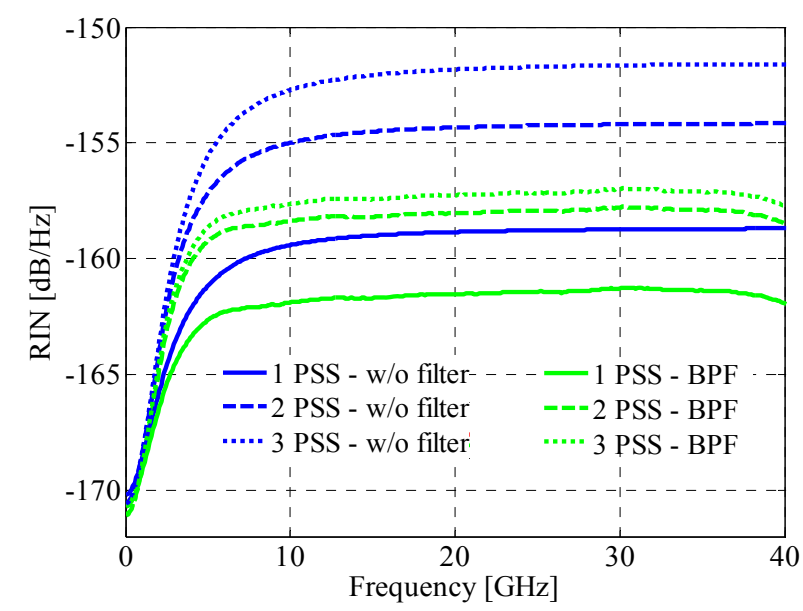

Fig. 6. Calculated RIN spectral density as a function of the number of the cascaded PSSs: (a) when no optical filtering is considered and (b) when using BPFs.

stages (RS) in-between individual PSS elements is necessary in order to restore the modulation sideband suppressed by previous filtering. RSs can conveniently be implemented by stand-alone SOAs, which exploit FWM for sideband regeneration [11]. Since the SOAs generates noise, this needs to be taken into account in noise studies.

Thanks to the modular nature of the numerical model depicted in Section II, simulations involving several PSSs with their respective RSs can be accomplished. The calculation of the RIN is performed in the frequency domain instead of the time domain, since this is found to provide faster computation times; this fact is of great importance when studying multiPSS-based structures. Moreover, problems related to coherent sampling are also avoided.

Fig. 6 shows the calculated RIN for the cases of no filter based and BPF-based schemes as a function of the number of PSSs. Simulations have been carried out by assuming identical physical parameters for all the SOAs, i.e. both for PSS and RS elements. Identical transfer functions for all the BPFs are considered.

The noise performance deterioration is noticeable when serially adding PSSs. Whatever the optical filtering scheme, the impact of connecting the third PSS is not as important as the fact of adding the second one. This is attributed to the larger gain saturation affecting the SOA located in the third stage compared with that belonging to the second one. The results derived for a single PSS can be extrapolated to the case of multi-PSS-based scheme. Besides, the cascading of PSSs yield extra flexibility for RF power variation tailoring purposes which can be exploited for improving the SNR [11].

\section{CONCLUSION}

A theoretical model to predict the RIN spectral density at the output of SOA-based MWP phase shifters has been presented. The model has shown very good agreement with experiments, showing its applicability to assess the RIN level even in presence of the microwave signal. Calculated data are consistent with experimental results when a non-dispersive optical filter is used. We have presented the way to alleviate 
the noise enhancement induced by FM-AM-noise conversion in dispersive optical filters. From the SNR results, the BPFbased scheme yields the optimum noise performance when considering one PSS. The results can be extrapolated to multiPSS-based structures. Suppression of the noise level by more than $5 \mathrm{~dB}$ has been reached in schemes based on band-pass optical filtering when three phase shifting stages are cascaded.

\section{REFERENCES}

[1] R. W. Boyd and D. J. Gauthier, "Controlling the Velocity of Light Pulses," Science, vol. 326, pp. 1074-1077, 2009.

[2] R. W. Boyd and D. J. Gauthier, "Slow and fast light," Prog. Optics, vol.43, pp. 497-530, 2002.

[3] J. Capmany and D. Novak, "Microwave photonics combines two worlds," Nature Photon., vol. 1, pp.319-330, 2007.

[4] J. Capmany, B. Ortega and D. Pastor, "A Tutorial on Microwave Photonic Filters,” J. Lightwave Technol., vol. 24, pp. 201-229, 2006.

[5] D. Dolfi, P. Joffre, J. Antoine, J-P. Huignard, D. Philippet and P. Granger, "Experimental demonstration of a phased-array antenna optically controlled with phase and time delays," Appl. Opt., vol. 35, pp. 5293-5300, 1996.

[6] C. Weil, S. Müller, P. Scheele, Y. Krivoshapka, P. Best, G. Lüssem and R. Jakoby, "Ferroelectric- and liquid crystal- tunable microwave phase shifter," Proc. $33^{\text {rd }}$ European Microwave Conf., vol. 3, pp. 1431-1434, 2003.

[7] J. Capmany, B. Ortega, D. Pastor and S. Sales, "Discrete-time optical processing of microwave signals," J. Lightwave Technol., vol. 23, pp. 702-723, 2005

[8] L. Thévenaz, "Slow and fast light in optical fibers," Nature Photon., vol. 2, pp. 474-481, 2008.

[9] T. Baba, "Slow Light in Photonic Crystals," Nature Photon., vol. 2, pp. 465-473, 2008.

[10] C. J. Chang and S. L. Chuang, "Slow and Fast Light in Semiconductor Quantum Well and Quantum-Dot Devices," J. Lightwave Technol., vol. 24, 4642-4654, 2006.

[11] W. Xue, S. Sales, J. Capmany and J. Mork, "Wideband $360^{\circ}$ microwave photonic phase shifter based on slow light in semiconductor optical amplifiers," Opt. Exp., vol. 18, pp. 6156-6163, 2010.

[12] J. Mork, P. Lunnemann, W. Xue, Y. Chen, P. Kaer and T. R. Nielsen, "Slow and fast light in semiconductor waveguide," Semicond. Sci. Technol., vol. 25, 083002, 2010.

[13] J. Mork, R. Kjær, M. Van der Poel and K. Yvind, "Slow light in a semiconductor waveguide at gigahertz frequencies," Opt. Exp., vol. 13, pp. 8136-8145, 2005.

[14] W. Xue, Y. Chen, F. Öhman, S. Sales and J. Mork, "Enhancing light slow-down in semiconductor optical amplifiers by optical filtering," Opt. Lett., vol. 33, no. 10, pp. 1084-1086, 2008.

[15] E. Shumakher, S. Ó. Dúill and G. Eisenstein, "Signal-to-noise ratio of a semiconductor-optical-amplifier-based optical phase shifter, Opt. Lett., vol. 34, no. 13, pp. 1940-1942, 2009.

[16] C. Cox, Analog Optical Links. U.K.: Cambridge Univ. Press, 2004

[17] J. Lloret, F. Ramos, J. Sancho, I. Gasulla, S. Sales and J. Capmany, "Noise Spectrum Characterization of Slow Light SOA-based Microwave Photonic Phase Shifters," Photon. Technol. Lett., vol. 22, no. 13, pp. 1005-1007, 2010

[18] S. Ó Dúill, E. Shumakher and G. Eisenstein, "Noise Properties of Microwave Phase Shifters based on Semiconductor Optical Amplifiers," J. Lightwave Technol., vol. 28, no. 5, pp. 791-797, 2010.

[19] I. Gasulla, J. Sancho, J. Lloret, S. Sales and J. Capmany, "Harmonic Distortion in Microwave Photonic Phase Shifters Based on Coherent Population Oscillations in SOAs," Photon. Technol. Lett., vol. 22, no. 12, pp. 899-901, 2010.

[20] P. Berger, J. Bourderionnet, M. Alouini, F. Bretenaker and D. Dolfi, "Theoretical Study of the Spurious-Free Dynamic Range of a Tunable Delay Line based on Slow Light in SOA", Opt. Exp., vol. 17, pp. 2058420597, 2009.

[21] M. Shtaif, B. Tromborg and G. Eisenstein, "Noise spectra of semiconductor optical amplifiers: Relation between semiclassical and quantum descriptions," IEEE J. Quant. Electr., vol. 34, no. 5, pp.869878, 1998.
[22] B. J. Eggleton, G. Lenz, N. Litchinitser, D. B. Patterson and R. E. Slusher, "Implications of Fiber Grating Dispersion for WDM Communications Systems," Photon. Technol. Lett., vol. 9, no. 10, pp. 1403-1405, 1997.

[23] J. Capmany, D. Pastor and B. Ortega, "RIN induced by out-band dispersion in fibre Bragg grating based add-drop multiplexers," Electr. Lett., vol. 35, no. 25, pp. 2220-2221, 1999.

[24] S. Ó Dúill, E. Shumakher and G. Eisenstein, "The role of optical filtering in microwave phase shifting," Opt. Lett., vol. 35, no. 13, pp. 2278-2280, 2010.

[25] S. Ó Dúill, E. Shumakher and G. Eisenstein, "Large-Signal Operation of a Semiconductor Optical Amplifier as a Phase-Shifting Element for Microwave Signals," Photon. Technol. Lett., vol. 21, no. 11, pp. 679681,2009. 